\title{
Triagem de glicolipídios em plantas medicinais
}

\author{
Beatriz G. Mendes ${ }^{1}$, Marcos J. Machado², Miriam Falkenberg ${ }^{3 *}$ \\ 'Programa de Pós-Graduação em Farmácia, Centro de Ciências da Saúde, Campus Trindade, Universidade \\ Federal de Santa Catarina, 88040-90, Florianópolis, SC, Brasil, \\ ${ }^{2}$ Departamento de Análises Clínicas, Centro de Ciências da Saúde, Campus Trindade, Universidade Federal de \\ Santa Catarina, 88040-900, Florianópolis, SC, Brasil, \\ ${ }^{3}$ Departamento de Ciências Farmacêuticas, Centro de Ciências da Saúde, Campus Trindade, Universidade Federal \\ de Santa Catarina, 88040-900, Florianópolis, SC, Brasil
}

\begin{abstract}
RESUMO: Os extratos obtidos pela maceração em $\mathrm{CHCl}_{3} / \mathrm{MeOH}(2: 1)$ de 8 amostras de espécies medicinais e 11 amostras comerciais de chás foram analisados por cromatografia em camada delgada (CCD). Glicolipídios foram detectados em todas as amostras, porém com diferenças qualiquantitativas. Para as plantas medicinais, a maior concentração de glicolipídios foi detectada em Lippia alba e Cymbopogon citratus, enquanto em amostras comerciais, o melhor perfil glicolipídico foi encontrado nos extratos de C. citratus e Baccharis trimera.
\end{abstract}

Unitermos: Glicolipídios, plantas medicinais, cromatografia em camada delgada.

\begin{abstract}
Screening of glycolipids in medicinal plants". The extracts obtained by maceration in $\mathrm{CHCl}_{3} / \mathrm{MeOH}(2: 1)$ of 8 medicinal plants and 11 commercial samples (tea bags) were analysed by thin-layer chromatography. Glycolipids were detected in all the samples, with qualitative and quantitative differences. For the medicinal plants, the highest concentrations were detected in Lippia alba and Cymbopogon citratus. For the commercial samples, the best glycolipidic profiles were found in C. citratus and Baccharis trimera extracts.
\end{abstract}

Keywords: Glycolipids, medicinal plants, thin-layer chromatography.

\section{INTRODUÇÃO}

Glicolipídios são macromoléculas amplamente distribuídas nos seres vivos, sendo comumente encontrados na parte externa das membranas celulares, tais como a membrana citoplasmática, mitocondrial, do retículo endotelial e dos cloroplastos (Kates, 1970). O termo glicolipídio designa compostos com uma ou mais unidades monossacarídicas, unidas através de ligações glicosídicas a uma molécula hidrofóbica, como acilglicerol ou ceramida (base esfingóide acilada), entre outras (IUPAC-IUB, 1999).

A variedade estrutural destes compostos é muito grande e conforme a natureza dos organismos, alguns tipos de glicolipídios são mais abundantes. Os do tipo ceramida (glicoesfingolipídios) são encontrados principalmente em tecidos de animais (Degroote; Wolthoorn; van Meer, 2004) enquanto aqueles contendo acilglicerol são amplamente distribuídos, sendo o principal tipo encontrado em vegetais (Morrison, 1986). Em plantas, são menos comuns os "fitoglicolipídios" (apresentando base esfingóide acilada), como os isolados de Mirabilis himalaica, usada na medicina tradicional Chinesa (Zhang et al., 1997). De plantas pertencentes à família Convolvulaceae foram isolados glicolipídios singulares, como as tricolorinas e orizabinas (Castelli et al., 2002).
Os glicoglicerolipídios são os maiores componentes da membrana tilacóide dos cloroplastos de vegetais superiores, e sua classificação está relacionada ao número ou tipo de açúcares ligados à molécula do glicerol, bem como ao número de ácidos graxos ligados à mesma. As classes mais comumente encontradas em plantas correspondem aos monogalactosildiacilgliceróis (MGDG), digalactosildiacilgliceróis (DGDG) e sulfoglicolipídios, estes últimos apresentando a unidade monossacarídica com grupo sulfato $\left(\mathrm{R}-\mathrm{OSO}_{3} \mathrm{H}\right)$ ou sulfonato ( $\left.\mathrm{R}_{-} \mathrm{SO}_{3} \mathrm{H}\right)$ (Ishizuka, 1997). Compostos com 3 ou 4 unidades de monossacarídio são consideravelmente mais raros.

Clinacanthus nutans (Burm.f.) Lindau (Acanthaceae) é uma planta amplamente utilizada na medicina popular da Tailândia para o tratamento de lesões herpéticas e outras afecções cutâneas. Recentemente, um estudo demonstrou que digalactosil- $\mathrm{e}$ trigalactosildiglicerol (respectivamente DGDG e TGDG) isolados a partir das folhas desta planta exibiram atividade antiviral contra Herpes simplex (Janwitayanuchit et al., 2003). Outro estudo com monogalactosildiacilglicerol (MGDG) isolado a partir do espinafre (Spinacia oleracea L.) demonstrou que esta substância é um potente inibidor do crescimento de uma linhagem de células humanas de câncer gástrico, inibindo também a atividade da DNApolimerase (Murakami et al., 2003a). 
Sulfoglicolipídios

como

o

sulfoquinovosildiacilglicerol (SQDG), isolado a partir da pteridófita Athyrium niponicum (Mizushina et al., 1998) e da alga vermelha Gigartina tenella (Ohta et al., 1999), apresentaram potente atividade inibitória da DNA-polimerase (Murakami et al., 2003a,b). SQDG e sulfoquinovosilmonoacilglicerol (SQMG) sintetizados demonstraram efeito antitumoral in vitro (Hanashima et al., 2001). Outro sulfoglicolipídio, o sulfoquinovosildipalmitoilglicerídio, isolado da alga marinha Dictyochloris fragrans Vischer ex Starr (Chlorophyta), demonstrou atividade como inibidor de receptores de P-selectinas, envolvidos em processos inflamatórios (Golik et al., 1997).

Atividade contra o vírus HIV foi demonstrada para sulfoglicolipídios isolados a partir de diferentes espécies de cianobactérias. Estes compostos inibiram eficiente e seletivamente a função da DNA-polimerase associada com a transcriptase reversa do HIV-1 (Reshef et al., 1997; Loya et al., 1998).

Frente à escassez de informações sobre estes compostos nas plantas de uso medicinal e uma vez que os glicolipídios de plantas podem ser fortes candidatos no desenvolvimento de novos fármacos para terapia do câncer, doenças virais e inflamatórias, entre outras, propusemo-nos, neste trabalho, realizar uma triagem de glicolipídios em extratos de algumas espécies medicinais comumente utilizadas na medicina popular, incluindo plantas in natura e amostras comerciais de chás, com vista à caracterização do perfil glicolipídico das espécies.

\section{MATERIAL E MÉTODOS}

Para a triagem de glicolipídios em plantas medicinais foram utilizadas folhas de oito espécies (tabela 1). O material vegetal foi coletado em Florianópolis, Santa Catarina, de espécimes identificados pelo Prof. Dr. Daniel de Barcellos Falkenberg, do Departamento de Botânica da UFSC. As exsicatas correspondentes encontram-se depositadas no Herbário FLOR, sob números 5381, 7491, 2584 a 2587, 29353 e 29383. Para a triagem em drogas vegetais dessecadas (chás) foram adquiridas 11 amostras comerciais de diferentes espécies (Tabela 2).

Plantas medicinais coletadas in natura foram secas em temperatura ambiente por 24 horas e moídas em moinho de facas. Todos os extratos foram preparados por maceração do material vegetal em $\mathrm{CHCl}_{3} / \mathrm{MeOH}$ (2:1) por 24 horas, na proporção $1: 5(\mathrm{~m} / \mathrm{v})$, filtrando-se e concentrando-se em evaporador rotatório a baixa pressão e temperatura inferior a $50^{\circ} \mathrm{C}$. Os resíduos foram pesados para o cálculo do rendimento em $\mathrm{g} / 100 \mathrm{~g}$ de planta. Os extratos secos foram ressuspendidos em $\mathrm{CHCl}_{3} / \mathrm{MeOH}$ 2:1 (v/v) de modo a obter solução na concentração de $5 \mu \mathrm{g} / \mu \mathrm{L}$. $20 \mu \mathrm{L}$ de cada solução foram aplicados em placas de gel de sílica do tipo HPTLC (Merck) e a análise cromatográfica foi realizada com eluente $\mathrm{CHCl}_{3} / \mathrm{MeOH} / \mathrm{H}_{2} \mathrm{O}$ 65:25:4 (v/v/v), borrifando-se com solução de orcinol-sulfúrico e aquecendo-se as placas a $100{ }^{\circ} \mathrm{C}$ para revelar as manchas. Foi utilizado padrão de digalactosildiacilglicerol (DGDG) de procedência Sigma. A caracterização dos glicolipídios foi baseada nos seus respectivos fatores de retenção (Rfs), de acordo com a literatura (Lepage, 1964; Machado, 1996; Machado et al., 1997). A intensidade das manchas orcinol-positivas

Tabela 1. Plantas medicinais in natura utilizadas na triagem de glicolipídios.

\begin{tabular}{|c|c|c|c|c|}
\hline Nome científico & Nome popular & Família & Alguns usos populares & \\
\hline $\begin{array}{l}\text { Baccharis trimera } \\
\text { (Less.) DC. }\end{array}$ & Carqueja & Asteraceae & $\begin{array}{l}\text { Digestivo, anti-helmíntico } \\
\text { (Simões et al., 1998) }\end{array}$ & \\
\hline $\begin{array}{l}\text { Bauhinia forficata } \\
\text { Link }\end{array}$ & Pata-de-vaca & Fabaceae & $\begin{array}{l}\text { Hipoglicemiante, } \\
\text { hipocolesterolemiante, } \\
\text { diurético } \\
\text { (Simões et al., 1998) }\end{array}$ & \\
\hline $\begin{array}{l}\text { Cymbopogon citratus } \\
\text { (DC.) Stapf }\end{array}$ & Capim-limão & Poaceae & $\begin{array}{l}\text { Ansiolítico, sedativo, } \\
\text { antifebril } \\
\text { (Simões et al., 1998) }\end{array}$ & \\
\hline $\begin{array}{l}\text { Lippia alba (Mill.) } \\
\text { N.E. Brown. }\end{array}$ & Erva-cidreira & Verbenaceae & $\begin{array}{l}\text { Ansiolítico, resfriados } \\
\text { (Simões et al., 1998) }\end{array}$ & \\
\hline $\begin{array}{l}\text { Maytenus ilicifolia } \\
\text { Mart. ex Reissek }\end{array}$ & $\begin{array}{l}\text { Espinheira-santa } \\
\text { (verdadeira) }\end{array}$ & Celastraceae & $\begin{array}{l}\text { Digestivo, gastrite } \\
\text { (Freire, 1996) }\end{array}$ & \\
\hline $\begin{array}{l}\text { Plectranthus barbatus } \\
\text { Andr. }\end{array}$ & Falso-boldo & Lamiaceae & $\begin{array}{l}\text { Problemas hepáticos e } \\
\text { intestinais } \\
\text { (Simões et al., 1998) }\end{array}$ & \\
\hline $\begin{array}{l}\text { Sorocea bonplandii } \\
\text { (Baill.) Burger, Lanj. } \\
\& \text { Boer }\end{array}$ & Espinheira-santa, mata-olho & Moraceae & $\begin{array}{l}\text { Digestivo, gastrite } \\
\text { (Pavan-Fruehauf, 2000) }\end{array}$ & \\
\hline $\begin{array}{l}\text { Zollernia ilicifolia } \\
\text { (Brongn.) Vogel }\end{array}$ & Espinheira-divina & Fabaceae & $\begin{array}{l}\text { Digestivo, gastrite } \\
\text { (Freire, 1996) }\end{array}$ & \\
\hline & & & $\begin{array}{r}\text { Rev. Bras. Farmacogn. } \\
\text { Braz J. Pharmacogn. } \\
\text { 16(4):out/dez. } 2006\end{array}$ & 569 \\
\hline
\end{tabular}


Tabela 2. Triagem de glicolipídios em amostras comerciais de chás.

\begin{tabular}{|c|c|c|c|}
\hline $\begin{array}{l}\text { Nome científico (conforme } \\
\text { embalagem) }\end{array}$ & Nome popular & $\begin{array}{l}\text { Família } \\
\text { (conforme embalagem) }\end{array}$ & Parte utilizada \\
\hline Achyrocline satureioides & Marcela & Asteraceae & Inflorescências \\
\hline Baccharis genistelloides & Carqueja & Asteraceae & Folhas \\
\hline Bauhinia forficata & Pata-de-vaca & Fabaceae & Folhas \\
\hline Cymbopogon citratus & Capim-cidreira & Poaceae & Folhas \\
\hline Cynara scolymus & Alcachofra & Asteraceae & Folhas \\
\hline Malva sylvestris & Malva & Malvacea & Folhas \\
\hline Matricaria recutita & Camomila & Asteraceae & Inflorescências \\
\hline Maytenus ilicifolia & Espinheira-santa & Celastraceae & Folhas \\
\hline Mentha piperita & Hortelã & Labiatae & Folhas \\
\hline Peumus boldus & Boldo-do-Chile & Monimiaceae & Folhas \\
\hline Pimpinella anisum & Erva-doce & Apiaceae & Frutos \\
\hline
\end{tabular}

foi avaliada visualmente e por densitometria com auxílio do software Scion Image (National Institut of Health), conforme descrito por Machado et al. (2001).

\section{RESULTADOS E DISCUSSÃO}

A triagem de espécies vegetais com potencial atividade biológica é uma ferramenta importante na busca de novos produtos naturais com potencial aplicação terapêutica (Funke; Melzig 2006; Leitão et al., 2006; Lima et al., 2006). Neste estudo, foram selecionadas espécies correspondentes às plantas mais citadas em levantamentos etnobotânicos realizados em Florianópolis e outras regiões do Brasil (Pagliarini, 1995; Freire, 1996; Pereira et al., 2004; Macedo; Ferreira 2004; Tôrres et al., 2005; Vendruscolo et al., 2005; Morais et al., 2005), sendo incluídas também diferentes espécies conhecidas pelo mesmo nome popular, como erva-cidreira (Lippia alba e Cymbopogon citratus) ou espinheira-santa (Maytenus ilicifolia, Sorocea bonplandii e Zollernia ilicifolia, as duas últimas também caracterizadas como adulterantes da primeira, conforme relatado por Alberton et al. (2003) e Coulaud-Cunha et al. (2004). Foram detectadas substâncias com reação positiva para orcinol-sulfúrico em todos os extratos testados.

O perfil cromatográfico dos extratos brutos das plantas medicinais é apresentado na Figura 1. A revelação desta placa foi feita com aquecimento rápido a $100{ }^{\circ} \mathrm{C}$ (suficiente para demonstrar as bandas dos compostos orcinol-positivos), com vistas a preservar o fundo claro da placa, para melhor contraste. Já para fins de densitometria e avaliação da intensidade das manchas orcinol-positivas,

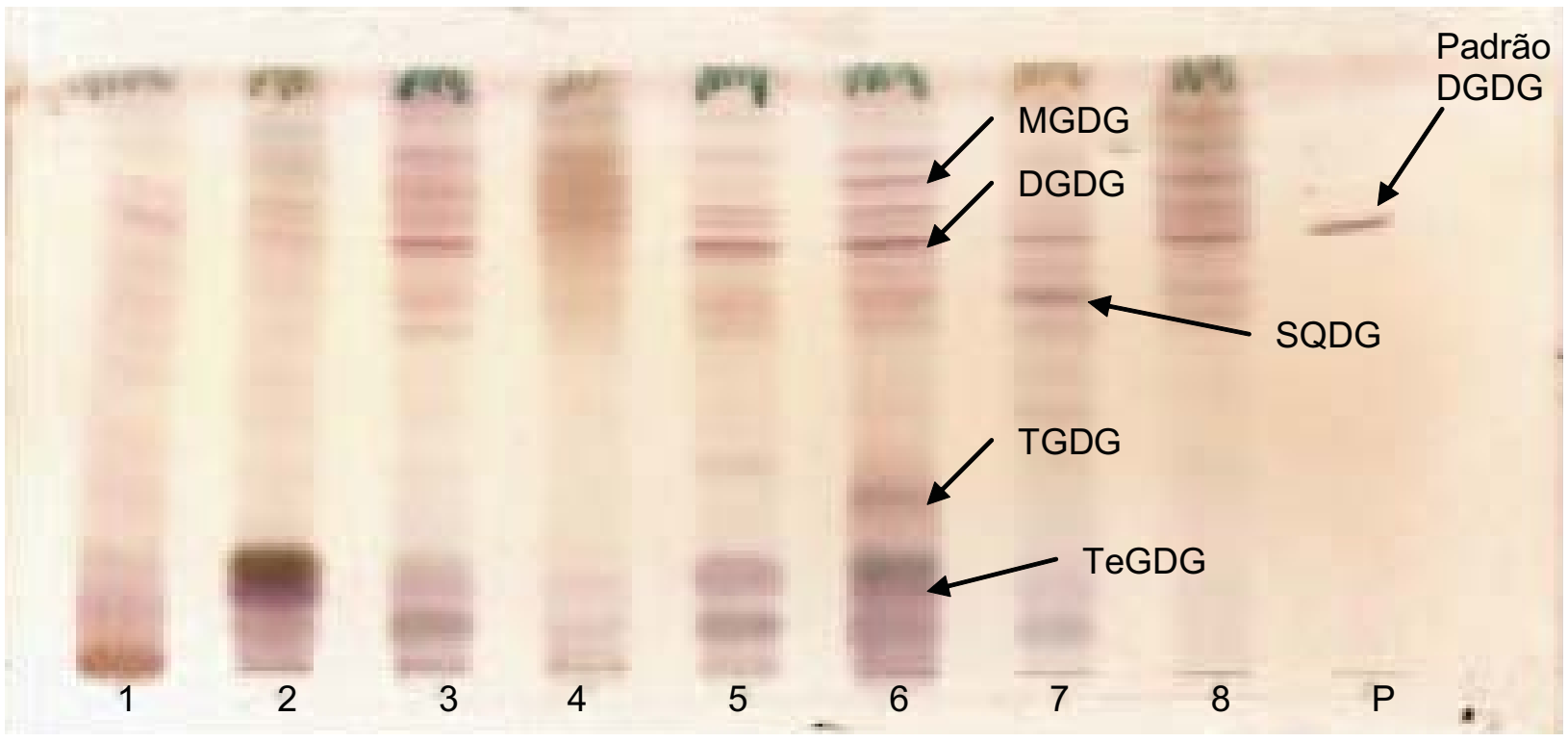

Figura 1. Cromatografia em camada delgada dos extratos brutos preparados com plantas medicinais in natura. Eluente: $\mathrm{CHCl}_{3} / \mathrm{MeOH} / \mathrm{H}_{2} \mathrm{O}$ (65:25:4); revelador: orcinol-sulfúrico/aquecimento. Da esquerda para direita: 1- Maytenus ilicifolia; 2-Sorocea bonplandii; 3-Zollernia ilicifolia; 4- Bauhinia forficata; 5- Lippia alba; 6- Cymbopogon citratus; 7- Baccharis trimera; 8-Plectranthus barbatus; P: Padrão de DGDG

(MGDG: monogalactosildiacilglicerol; DGDG: digalactosildiacilglicerol; TGDG: trigalactosildiacilglicerol; TeGDG: tetragalactosildiacilglicerol; SQDG: sulfoquinovosildiacilglicerol). 
Tabela 3. Valores de Rf das manchas orcinol-positivas (figura 1) e os correspondentes tipos de glicolipídios a elas atribuídos, com base na literatura (*=valores de $\mathrm{Rf}$ não foram encontrados na literatura consultada).

\begin{tabular}{|c|c|c|}
\hline $\begin{array}{l}\text { Rf manchas } \\
\text { orcinol-positivas (figura } 1 \text { ) }\end{array}$ & $\begin{array}{l}\text { Tipo de glicolipídio proposto e } \\
\text { faixa de Rf esperada }\end{array}$ & Literatura base para atribuição \\
\hline 0,8 & $\mathrm{MGDG}=0,77-0,83$ & Lepage, 1964; Schwertner; Biale, 1973 \\
\hline 0,71 & DGDG $=0,62-0,7$ & $\begin{array}{l}\text { Lepage, 1964; Machado et al., 1997; } \\
\text { Lhullier, 2005; Lhullier et al., } 2006\end{array}$ \\
\hline 0,61 (reação atípica) & Sulfolipídio/SQDG = 0,42-0,46 & Lepage, 1964; Sassaki et al., 2001 \\
\hline 0,28 & trigalactosildiacilglicerol $(\mathrm{TGDG})=0,35$ & Sassaki et al., 1999 \\
\hline 0,15 & tetragalactosildiacilglicerol (TeGDG) & $*$ \\
\hline
\end{tabular}

as placas foram submetidas a aquecimento mais prolongado.

$\mathrm{Na}$ análise cromatográfica foi possível, a partir do valor de Rf observado para o padrão de digalactosildiacilglicerol (DGDG) e de dados da literatura, sugerir a presença também de outros tipos de glicolipídios (Tabela 3).

Para o glicolipídio em $\mathrm{Rf}=0,61$, que apresentou reação atípica para orcinol-sulfúrico, foi cogitada a possibilidade de tratar-se de um sulfolipídio. Reação semelhante foi verificada por Sassaki et al. (2001) para sulfoglicolipídiosdeDictyonemaglabratum (comunicação pessoal). Segundo Ishizuka (1997), tal grupo de glicolipídios pode apresentar variações conforme o átomo de enxofre ligar-se a carbono ou oxigênio, além daquelas relacionadas com o número e posição de grupos funcionais contendo enxofre. Sulfolipídios do tipo sulfoquinovosildiacilglicerol (SQDG), com grupos acila esterificando dois grupos hidroxila do glicerol, são comumente descritos na literatura como "plant sulfolipid” (Quasney et al., 2001; Sassaki et al., 2001). Foram encontradas poucas referências de valores de $\mathrm{Rf}$ no sistema eluente empregado (tabela 3); Ishizuka (1997) discute o comportamento cromatográfico de vários outros sulfoglicolipídios, inclusive em outros sistemas cromatográficos considerados mais adequados para sulfoglicolipídos mais complexos, mas não faz menção a valores de $\mathrm{Rf}$.

A diferente mobilidade cromatográfica dos vários tipos de glicolipídios no sistema eluente recomendado para glicolipídios neutros está relacionada com o número de açúcares ligados às moléculas, a natureza dos ácidos graxos presentes, bem como o tipo de álcool ou base aminada presente (Morrison, 1986). O padrão aplicado de DGDG apresentou Rf 0,71, valor superior ao relatado por Lepage (1964) utilizando placas preparadas no próprio laboratório. Considerando-se que as placas de sílica para HPTLC utilizadas neste trabalho têm granulometria diferente das placas usadas por aqueles autores e que outras variáveis, como a umidade relativa do ar, também podem interferir nos valores de $\mathrm{Rf}$ em análises de CCD, é possível sugerir que as manchas em valores de Rf decrescentes visualizadas nos cromatogramas correspondam à seqüência: monogalactosil-, digalactosildiacilglicerol, sulfoglicolipídio (com estrutura sulfoquinovosil) e glicolipídios mais polares (tri- e tetragalactosildiacilglicerol).

Considerando que a porção estrutural correspondente aos ácidos graxos dos glicolipídios detectados nos extratos pode apresentar diferença em relação àquela dos glicolipídios cujos valores de $\mathrm{Rf}$ são citados na literatura, para fins de triagem inicial a classificação em função da polaridade conferida pelas unidades de açúcar pode ser considerada válida. Devese também ressaltar que variações nos ácidos graxos de glicolipídios foram relatadas para diferentes espécies de algas (Xue et al., 2002; Dembitsky et al., 2003) e liquens (Sassaki et al., 2001, que discute amplamente este aspecto, inclusive em relação a plantas).

Os rendimentos dos extratos lipídicos das plantas coletadas in natura, bem como a interpretação da sua análise cromatográfica, estão resumidos na tabela 4. Pode-se observar que todos os extratos investigados apresentam glicolipídios, porém com algumas diferenças quali-quantitativas. Na avaliação do rendimento dos extratos brutos obtidos de plantas medicinais frescas, pode-se observar que houve diferenças de rendimento de até 10 vezes. Assim, o extrato de Lippia alba apresentou rendimento de $11,3 \%$, enquanto para Plectranthus barbatus orendimento foide $1,1 \%$. Naliteraturapesquisada foram encontrados relatos de teores variáveis, dependendo da espécie e da parte da planta testada. Asilbekova (2004) relatou para frutos de Capsicum annuum (Solanaceae) a ocorrência de maior teor de glicolipídios na polpa $(2,45 \%)$ do que nas sementes $(1,8 \%)$, mas estes valores correspondem a determinação espectrofotométrica em frações purificadas cromatograficamente, enquanto os valores apresentados nas tabelas 1 e 2 correspondem a determinação gravimétrica do extrato lipídico total. Para Veronica beccabunga (Scrophulariaceae) os rendimentos de extrato lipídico total variaram de $1,25 \%$ para as flores a $0,14 \%$ para os caules (Rozentsvet; Bosenko, 2002), mas estes dados referem-se a extração com a mistura $\mathrm{CHCl}_{3} / \mathrm{MeOH} / \mathrm{H}_{2} \mathrm{O}(1: 2: 0,8)$. Em espécies da família Convolvulaceae, diversos estudos apontaram teor maior de glicolipídios em raízes (5\%), seguido por sementes (3$5 \%$ ) e folhas (2\%), conforme revisão de Tulloch (1986).

\begin{tabular}{r|c}
\hline Rev. Bras. Farmacogn. & 571 \\
Braz J. Pharmacogn. & \\
16(4):out/dez. 2006 &
\end{tabular}


Tabela 4. Rendimento dos extratos brutos das espécies medicinais testadas e tipos de glicolipídios detectados nos mesmos. A intensidade das manchas com reação para orcinol-sulfúrico está representada conforme a convenção: ND $=$ não detectado,$+=$ fraca intensidade,$++=$ média intensidade $\mathrm{e}+++$ forte intensidade.

\begin{tabular}{clcccccc}
\hline Número & Espécie & Rend. $\%$ & MGDG & DGDG & TGDG & TeGDG & SQDG \\
\hline $\mathbf{1}$ & Maytenus ilicifolia & 6,3 & + & + & + & ++ & + \\
$\mathbf{2}$ & Sorocea bonplandii & 5,2 & + & + & + & +++ & + \\
$\mathbf{3}$ & Zollernia ilicifolia & 5,6 & + & +++ & ++ & ++ & + \\
$\mathbf{4}$ & Bauhinia forficata & 2,7 & + & + & + & + & + \\
$\mathbf{5}$ & Lippia alba & 11,3 & ++ & +++ & + & +++ & ++ \\
$\mathbf{6}$ & Cymbopogon citratus & 5,6 & +++ & ++++ & ++ & +++ & ++ \\
$\mathbf{7}$ & Baccharis trimera & 9,1 & + & +++ & + & + & ++++ \\
$\mathbf{8}$ & Plectranthus barbatus & 1,1 & +++ & ++++ & + & + & ++ \\
\hline
\end{tabular}

Outro fato observado é que o conteúdo glicolipídico não teve relação com o rendimento: plantas que obtiveram extrato bruto com alto rendimento não necessariamente apresentaram maiores concentrações de glicolipídios individuais. $\mathrm{O}$ extrato de Baccharis trimera com rendimento de 9,1\% apresentou menores concentrações de diversos glicolipídios em relação ao extrato de Cymbopogon citratus, cujo rendimento foi de $5,6 \%$.

Em relação ao conteúdo glicolipídico, pode-se observar que de modo geral os resultados obtidos estão de acordo com o descrito por Kates (1986) para tecidos fotossintéticos, onde MGDG e DGDG são encontrados em maior concentração. Em termos de abundância, em plantas, glicosildiacilgliceróis são geralmente presentes em maior quantidade. Com exceção dos extratos de Bauhinia forficata, Maytenus ilicifolia e da espécie adulterante desta última, Sorocea bonplandii, todos os demais apresentaram grandes concentrações de DGDG. Cymbopogon citratus e a outra adulterante da espinheirasanta, Zollernia ilicifolia, apresentaram ainda manchas de média intensidade (++) em Rf atribuível a TGDG.

Tabela 5. Rendimento dos extratos brutos obtidos a partir de amostras comerciais e glicolipídios detectados nos mesmos $(+\mathrm{a}++++$ indica a intensidade de reação ao orcinol-sulfúrico).

\begin{tabular}{|c|c|c|c|c|c|c|}
\hline $\begin{array}{l}\text { Espécie (conforme indicação da } \\
\text { embalagem) }\end{array}$ & $\begin{array}{l}\text { Rend. } \\
\mathrm{g} / 100 \mathrm{~g} \\
\text { planta }\end{array}$ & MGDG & DGDG & TGDG & TeGDG & $\mathrm{SQDG}$ \\
\hline Maytenus ilicifolia & 31,07 & + & + & + & ++++ & + \\
\hline Mentha piperita & 8,82 & ++ & + & + & + & + \\
\hline Peumus boldus & 27,31 & + & ++++ & + & + & + \\
\hline Cymbopogon citratus & 16,47 & + & ++++ & + & + & ++ \\
\hline Matricaria recutita & 30,05 & + & + & + & ++ & + \\
\hline Baccharis genistelloides & 23,14 & +++ & ++++ & ++++ & +++ & ++ \\
\hline Pimpinella anisum & 24,76 & + & + & + & + & + \\
\hline Bauhinia forficata & 38,78 & + & + & + & +++ & ++ \\
\hline Cynara scolymus & $\mathrm{Nd}^{*}$ & + & + & + & + & + \\
\hline Achyrocline satureioides & 20,91 & + & + & + & + & + \\
\hline Malva sylvestris & 5,12 & + & + & + & + & + \\
\hline
\end{tabular}

*Nd: Não foi possível calcular o rendimento para essa amostra

Tabela 6. Comparação entre rendimento e conteúdo lipídico de amostras in natura (IN) e comerciais (C) da mesma espécie (+ a ++++ indicam a intensidade de reação ao orcinol-sulfúrico, ND = não detectado).

\begin{tabular}{|c|c|c|c|c|c|c|}
\hline Amostra & Rend. & MGDG & DGDG & TGDG & TeGDG & SQDG \\
\hline M. ilicifolia $\mathrm{IN}$ & 6,3 & + & + & + & + & + \\
\hline M. ilicifolia $\mathrm{C}$ & 31,07 & + & + & + & ++++ & ND \\
\hline Baccharis IN & 9,1 & + & + & + & +++ & + \\
\hline Baccharis C & 23,14 & +++ & ++++ & ++++ & +++ & ND \\
\hline B. forficata IN & 2,7 & + & ++++ & ++++ & +++ & + \\
\hline B. forficata $\mathrm{C}$ & 38,78 & + & + & + & +++ & ND \\
\hline C. citratus IN & 5,6 & ++++ & ++++ & ++++ & + & ++++ \\
\hline C. citratus $\mathrm{C}$ & 16,47 & + & ++++ & + & + & ND \\
\hline
\end{tabular}


Destaca-se, ainda, a alta concentração de glicolipídios em Rf compatível com TeGDG nos extratos de Sorocea bonplandii, Lippia alba e Cymbopogon citratus. Analisando glicolipídios em sementes de Nigella sativa L. e Coriandrum sativum L., Ramadan e Mörsel (2002) encontraram perfis cromatográficos similares para estas espécies, com prevalência do componente DGDG, sendo sulfolipídios detectados em baixa concentração apenas na primeira; tri- e tetradiacilgliceróis não foram detectados. Rozentsvet e Bosenko (2002) também constataram predomínio de MGDG em relação a MGDG em folhas, raízes e flores de Veronica beccabunga, sendo que os caules continham DGDG em concentração ligeiramente superior. Os extratos das flores e raízes desta espécie continham maior teor de SQDG em relação aos caules e folhas.

As amostras comerciais utilizadas na triagem foram processadas e analisadas da mesma maneira que as plantas medicinais. Também neste grupo observouse uma grande variação no perfil de glicolipídios e no rendimento dos extratos brutos (Tabela 5).

Para quatro das espécies testadas que foram coletadas in natura foi possível a aquisição de amostras comerciais com identificação correspondente. Cabe destacar que Baccharis genistelloides é uma sinonímia de Baccharis trimera (Less.) DC., entretanto, na tabela 4 a sinonímia foi utilizada, por ser o nome citado na embalagem. Em todos os casos houve diferenças significativas no perfil cromatográfico e/ou no rendimento de glicolipídios para a mesma (suposta) espécie (Tabela 6). Seriam esperadas diferenças na composição química em função do local e época de coleta, bem como devido a diferenças no processo de secagem, entre outros fatores. $\mathrm{O}$ estresse hídrico a que podem ser submetidos os vegetais em crescimento influencia o conteúdo de glicolipídios: algumas espécies como trigo e cevada apresentaram redução de MGDG e DGDG (Chetal et al., 1982), enquanto soja e girassol tiveram aumento desses glicolipídios, em comparação aos controles, após estresse hídrico (Navari-Izzo; Vangioni; Quartacci, 1990). Outro fator relacionado ao ambiente é a possibilidade de plantas expostas a altos níveis de poluição acumularem outros tipos de metabólitos, como os arsenolipídios, que já foram detectados em inúmeras espécies (Dembitsky; Levitsky, 2004).

Para todas as amostras in natura, o rendimento obtido foi menor em relação às correspondentes amostras comerciais, o que se poderia explicar, pelo menos em parte, devido ao processo mais drástico de secagem a que drogas vegetais processadas industrialmente costumam ser submetidas. De acordo com a tabela 6, pode-se observar grande variação no conteúdo glicolipídico individual. A amostra comercial de carqueja e a amostra in natura de Cymbopogon citratus foram as que apresentaram manchas mais intensas de glicolipídios.

Para amostras comerciais de Matricaria recutita e Achyrocline satureioides, das quais se utilizaram as inflorescências para o teste, obteve-se um rendimento de extrato bruto superior a $20 \%$, que foi em alguns casos maior que aqueles observados para plantas das quais se utilizaram folhas. O mesmo foi observado para amostra de Pimpinella anisum, constituída por frutos. Isto pode ser explicado pelo fato de que os glicolipídios são encontrados não apenas em tecidos fotossintéticos, mas também naqueles não-fotossintéticos, como sementes, troncos, frutos, vagem (leguminosas), entre outros (Kojima et al., 1991).

A detecção de compostos com reação orcinolpositiva, com características de glicolipídios, em diferentes espécies de plantas medicinais abre perspectivas para futuros trabalhos de isolamento, elucidação e avaliação de potenciais atividades biológicas.

\section{REFERENCIAS}

Alberton MD, Falkenberg DB, Falkenberg MB 2002. Análise cromatográfica de fitoterápicos à base de espinheirasanta (Maytenus ilicifolia). Rev Bras Farmacogn 12: 11-13.

Asilbekova, DT 2004. Glycolipids from Capsicum annuum. Chem Nat Compd 40: 115-117.

Castelli MV, Cortes JCG, Escalante AM, Bah M, PeredaMiranda R, Ribas JC, Zacchina SA 2002. In vitro inhibition of 1,3-beta-glucan synthase by glycolipis from convolvulaceous species. Planta Med 68: 739742.

Chetal S, Wagle DS, Nainawatee HS 1982. Alterations in glycolipids of wheat and barley leaves under water stress. Phytochemistry 21: 51-53.

Coulaud-Cunha S, Oliveira RS, Waissmann W 2004. Vensa livre de Sorocea bomplandii Bailon como espinheirasanta no município de Rio de Janeiro-RJ. Rev Bras Farmacogn 14(Supl. 1): 51-53.

Degroote S, Wolthoorn J, van Meer G 2004. The cell biology of glycosphingolipids. Semin Cell Dev Biol 15: 375387.

Dembitsky VM, Levitsky DO 2004. Arsenolipids. Progr Lipid Res 43: 403-448.

Dembitsky VM, Rezankova H, Rezanka T, Hanus LO 2003. Variability of the fatty acids of the marine green algae belonging to the genus Codium. Biochem Syst Ecol 31: 1125-1145.

Freire RM 1996. Levantamento das plantas de uso medicinal nas comunidades do Canto dos Araçás e Costa da Lagoa, Ilha de Santa Catarina, Florianópolis, SC. Trabalho de Conclusão do Curso de Bacharelado em Ciências Biológicas, Universidade Federal de Santa Catarina.

Funke I, Melzig MF 2006. Traditionally usad plants in diabetes therapy - phytotherapeutics as inhibitors of $\alpha$-amylase activity. Rev Bras Farmacogn 16: 1-5.

Golik J, Dickey JK, Todderud G, Lee D, Alford J, Huang S, Klohr S, Eustice D, Aruffo A, Agler ML 1997. Isolation and structure determination of sulfonoquinovosyl dipalmitoyl glyceride, a P-selectine receptor inhibitor from the alga Dictyochloris fragrans. J Nat Prod 60: 387-389.

Hanashima S, Mizushina Y, Yamazaki T, Ohta K, Takahashi S, 
Sahara H, Sakaguchi K, Sugawara F 2001. Synthesis of sulfoquinovosylacylglycerols, inhibitors of Eukaryotic DNA Polymerase $\alpha$ and $\beta$. Bioorg Med Chem 9: 367-376.

Ishizuka I 1997. Chemistry and functional distribution of sulfoglycolipids. Prog Lipid Res 36: 245-319.

IUPAC-IUB Joint Commission on Biochemical Nomenclature (JCBN) 1999. Nomenclature of Glycolipids. $J \mathrm{Mol}$ Biol 286: 963-970.

Janwitayanuchit W, Suwanborirux K, Patarapanich C, Pummangura S, Lipipun V, Vilaivan T 2003. Synthesis and anti-herpes simplex viral activity of monoglycosyl diglycerides. Phytochemistry 64: 1253-1264.

Kates M 1970. Plant phospholipids and glycolipids. Adv Lipid Res 8: 225-265.

Kates M 1986. Techniques of lipidology: isolation, analysis and identification of lipids. 2.ed. New York: Elsevier.

Kojima M, Kimura H, Ohnishi M, Fujino Y, Ito S 1991. Distribution and characterization of diglycosyldiacylglycerol isomers with different anomeric configurationinhigherplants. Phytochemistry 30: $1165-1168$.

Leitão SG, Castro O, Fonseca EM, Julião LS, Tavares ES, Leo RRT, Vieira RC, Oliveira DR, Leitão GG, Martino V, Sulsen V, Barbosa YAG, Pinheiro DPG, Silva PEA, Teixeira DF, Lourenço MCS 2006. Screening of Central and South American plant extracts for antimycobacterial activity by the Alamar Blue test. Rev Bras Farmacogn 16: 6-11.

Lepage M 1964. The separation and identification of plant phospholipids and glycolipids by two-dimensional thin-layer chromatography. $J$ Chromatogr 13: 99103.

Lhullier C 2005. Triagem de macroalgas bênticas do litoral de santa catarina biomonitorada pelo ensaio de letalidade para larvas de Artemia salina e investigação fitoquímica de Pterocladiella capillacea. Florianópolis, 87p. Dissertação de mestrado - Programa de Pósgraduação em Farmácia, Universidade Federal de Santa Catarina.

Lhullier C, Horta PA, Falkenberg M 2006. Avaliação de extratos de macroalgas bênticas do litoral catarinense utilizando o teste de letalidade para Artemia salina. Rev Bras Farmacogn 16: 158-163.

Lima MRF, Ximenes CPA, Luna JS, Sant'Ana AEG 2006. The antibiotic activity of some Brazilian medicinal plants. Rev Bras Farmacogn 16: 300-306.

Loya S, Reshef V, Mizrachi E, Silberstein C, Rachamim Y, Carmeli S, Hizi A 1998. The inhibitor of the reverse transcriptase of HIV-1 by the natural sulfoglycolipids from cyanobacteria: contribution of the different moieties to their high potency. J Nat Prod 61: 891895.

Macedo M, Ferreira AR 2004. Plantas hipoglicemiantes utilizadas por comunidades tradicionais na Bacia do Alto Paraguai e Vale do Guaporé, Mato Grosso-Brasil. Rev Bras Farmacogn 14(Supl. 1): 45-47.

Machado MJ 1996. Glicolipídios de Ramalina celastri: isolamento e caracterização estrutural. Curitiba, $174 \mathrm{p}$. Tese de Doutorado - Programa de Pós-Graduação em Bioquímica, Universidade Federal do Paraná.

Machado MJ, Guerrini M, Gorin PAJ, Torri G, Iacomini M 1997. A galactosphingolipid from the lichen Ramalina celastri. Phytochemistry 45: 651-653.

Machado MJ, Borges FP, Falkenberg MB, Lima LM, Rovaris ML, Sassaki G, Iacomini M 2001. Determinação de glicolipídios em urinas. NewsLab 44: 98-112.

Mizushina Y, Watanabe I, Ohta K, Takemura M, Sahara H, Takahashi N, Gasa S, Sugawara F, Matsukage A, Yoshida S, Sakaguchi K 1998. Studies on inhibitors of mammalian DNA polymerase $\alpha$ and $\beta$ : sulfolipids from a pteridophyte, Athyrium niponicum. Biochem Pharmacol 55: 537-541.

Morais SM, Dantas JDP, Silva ARA, Magalhães EF 2005. Plantas medicinais usadas pelos índios Tapebas do Ceará. Rev Bras Farmacogn 15: 169-177.

Morrison IM 1986. Glycolipids. In: Chaplin MF, Kennedy, JF (Eds.) Carbohydrate analysis: a practical approach. Oxford: IRL, p.205-221.

Murakami C, Kumagai T, Hada T, Kanekazu U, Nakazawa S, Kamisuki S, Maeda N, Xu X, Yoshida H, Sugawara F, Sakaguchi K, Mizushina Y 2003a. Effects of glycolipids from spinach on mammalian DNA polymerases. Biochem Pharmacol 65: 259-267.

Murakami C, Yamazaki T, Hanashima S, Takahashi S, Takemura M, Yoshida S, Ohta K, Yoshida H, Sugawara F, Sakaguchi K, Mizushina Y 2003b. A novel DNA polymerase inhibitor and a potent apoptosis inducer: 2 mono-O-acyl-3-O-( $\alpha$-D-sulfoquinovosyl)-glyceride with stearic acid. BBA-Proteins Proteom 1645: 72 80 .

Navari-Izzo F, Vangioni N, Quartacci MF 1990. Lipids of soybean and sunflower seedlings grown under drought conditions. Phytochemistry 29: 2119-2123.

Ohta K, Mizushina Y, Hirata N, Takemura M, Sugawara F, Matsukage A, Yoshida S, Sakaguchi K 1999. Action of a new mammalian DNA polymerase inhibitor, sulfoquinovosyl diacylglycerol. Biol Pharm Bull 22: 111-116

Pagliarini WFM 1995. Levantamento das plantas de uso medicinal no Distrito de Ribeirão da Ilha, Florianópolis, SC. Trabalho de Conclusão do Curso de Bacharelado em Ciências Biológicas, Universidade Federal de Santa Catarina.

Pavan-Fruehauf S 2000. Plantas Medicinais de Mata Atlântica - manejo sustentado e amostragem. São Paulo: Annablume/Fapesp.

Pereira RC, Oliveira MTR, Lemos GCS 2004. Plantas utilizadas como medicinais no município de Campos de Goytacazes - RJ. Rev Bras Farmacogn 14 (Supl. 1): 37-40.

Quasney ME, Carter LE, Oxford C, Watkins SM, Gershwin ME, German JB 2001. Inhibition of proliferation and induction of apoptosis in SNU-1 human gastric cancer cells by the plant sulfolipid, sulfoquinovosyldiacylglycerol. J Nutrit Biochem 12: 310-315.

Ramadan MF, Mörsel J-T 2002. Analysis of glycolipids from black cumin (Nigella sativa L.), coriander (Coriandrum sativum L.) and niger (Guizotia abyssinica Cass.) oilseeds. Food Chem 80: 197-204.

Reshef V, Mizrachi E, Maretzki T, Silberstein C, Loya S, Hizi A, Carmeli S 1997. New acylated sulfoglycolipids and digalactolipids and related known glycolipids from cyanobacteria with a potential to inhibit the reverse transcriptase of HIV-1. J Nat Prod 60: 1251-1260. 
Rozentsvet OA, Bosenko EN 2002. Characteristics of lipids from individual organs of Veronica beccabunga. Chem Nat Compd 38: 521-523.

Sassaki GL, Machado MJ, Tischer CA, Gorin PAJ, Iacomini M 1999. Glycosyldiacylglycerolipids from the lichen Dictyonema glabratum. J Nat Prod 62: 844-847.

Sassaki GL, Gorin PAJ, Tischer CA, Iacomini M 2001. Sulfonoglycolipids from the lichenized basidiomycete Dictyonema glabratum: isolation, NMR, and ESI-MS approaches. Glycobiology 11:345-351.

Schwertner HA, Biale JB 1973. Lipid composition of plant mitochondria and of chloroplasts. J Lipid Res 14: 235239.

Simões CMO, Mentz LA, Schenkel EP, Irgang BE, Stehmann JR 1998. Plantas da medicina popular no Rio Grande do Sul. 4.ed. Porto Alegre: Editora da UFRGS.

Tôrres AR, Oliveira RAG, Diniz MFFM, Araújo EC 2005. Estudo sobre o uso de plantas medicinais em crianças hospitalizadas da cidade de João Pessoa: riscos e benefícios. Rev Bras Farmacogn 15: 373-380.

Tulloch AP 1986. Glycosides of hydroxy fatty acids. In: Kates M (Ed) Techniques of lipidology: isolation, analysis and identification of lipids. 2.ed. New York: Elsevier, p.463-487.

Vendruscolo GS, Rates SMK, Mentz LA 2005. Dados químicos e farmacológicos sobre as plantas utilizadas como medicinais pela comunidade do bairro Ponta Grossa, Porto Alegre, Rio Grande do Sul. Rev Bras Farmacogn 15: 361-372.

Xue C, Hu Y, Saito H, Zhang Z, Li Z, Cai Y, Ou C, Lin H, Imbs AB 2002. Molecular species composition of glycolipids from Spirulina platensis. Food Chem 77: 9-13.

Zhang G-L, Xing Q-Y, Zhang M-Z 1997. Glycolipids from Mirabilis himalaica. Phytochemistry 45: 1213-1215. 